\title{
Computing the long-distance contribution to the kaon mixing parameter $\varepsilon_{K}$
}

\author{
Norman Christ ${ }^{* \dagger}$ \\ Department of Physics \\ Columbia University, USA \\ E-mail: nhc@phys.columbia.edu
}

\section{RBC and UKQCD collaborations}

The largest contribution to the $\mathrm{CP}$ violating $K_{L}-K_{S}$ mixing parameter $\varepsilon_{K}$ comes from second order weak interactions at short distances and can be accurately determined by a combination of electroweak perturbation theory and the calculation of the parameter $B_{K}$ from lattice QCD. However, there is an additional long distance contribution to $\varepsilon_{K}$ which is estimated to be of order $5 \%$. Here recently introduced lattice techniques for computing the long-distance component of the $K_{L}-K_{S}$ mass difference are generalized to this long-distance contribution to $\varepsilon_{K}$.

XXIX International Symposium on Lattice Field Theory

July 10 - 162011

Squaw Valley, Lake Tahoe, California

\footnotetext{
*Speaker.

${ }^{\dagger}$ This work was supported in part by U.S. DOE grant DE-FG02-92ER40699
} 


\section{Introduction}

Some of the most stringent tests of the standard model and most promising windows into possible new physics come from process which occur at second order in the Fermi constant $G_{F}$. These include the standard model prediction for indirect CP violation in the $K^{0}-\bar{K}^{0}$ system and the bound on interactions which change strangeness by two units coming from the small $K_{L}-K_{S}$ mass difference. Such $O\left(G_{F}^{2}\right)$ amplitudes involve the exchange of two intermediate bosons which requires that each pair of vertices joined by such a $W^{ \pm}$exchange are separated by a short distance on the order of $1 / m_{W}$. However, the spatial separation between these two pairs of vertices need not be small, and "long distance" separations of the order of $1 / \Lambda_{\mathrm{QCD}}$ are possible.

This is well illustrated by the familiar Wigner-Weisskopf formula describing the time evolution of the $K^{0}-\bar{K}^{0}$ system:

$$
i \frac{d}{d t}\left(\begin{array}{c}
K^{0} \\
\bar{K}^{0}
\end{array}\right)=\left\{\left(\begin{array}{cc}
M_{00} & M_{0 \overline{0}} \\
M_{\overline{0} 0} & M_{\overline{00}}
\end{array}\right)-\frac{i}{2}\left(\begin{array}{cc}
\Gamma_{00} & \Gamma_{0 \overline{0}} \\
\Gamma_{\overline{0} 0} & \Gamma_{\overline{00}}
\end{array}\right)\right\}\left(\begin{array}{c}
K^{0} \\
\bar{K}^{0}
\end{array}\right)
$$

where the $2 \times 2$ matrices $M$ and $\Gamma$ are given by:

$$
\begin{aligned}
\Gamma_{i j} & =2 \pi \sum_{\alpha} \int_{2 m_{\pi}}^{\infty} d E\left\langle i\left|H_{W}\right| \alpha(E)\right\rangle\left\langle\alpha(E)\left|H_{W}\right| j\right\rangle \delta\left(E-m_{K}\right) \\
M_{i j} & =\sum_{\alpha} \mathscr{P} \int_{2 m_{\pi}}^{\infty} d E \frac{\left\langle i\left|H_{W}\right| \alpha(E)\right\rangle\left\langle\alpha(E)\left|H_{W}\right| j\right\rangle}{m_{K}-E} .
\end{aligned}
$$

We are using the subscripts 0 and $\overline{0}$ to represent the $K^{0}$ and $\bar{K}^{0}$ states. The generalized sum over $\alpha$ and integral over the energy represents the sum over a complete set of energy eigenstates normalized as $\left\langle\alpha^{\prime}\left(E^{\prime}\right) \mid \alpha(E)\right\rangle=\delta\left(E^{\prime}-E\right) \delta_{\alpha^{\prime} \alpha}$. These two matrices determine the two important quantities, $m_{K_{S}}-m_{K_{L}}$ and $\varepsilon_{K}$ :

$$
\begin{aligned}
m_{K_{S}}-m_{K_{L}} & =2 \operatorname{Re}\left\{M_{0 \overline{0}}\right\} \\
\varepsilon_{K} & =\frac{i}{2}\left\{\frac{\operatorname{Im} M_{0 \overline{0}}-\frac{i}{2} \operatorname{Im} \Gamma_{0 \overline{0}}}{\operatorname{Re} M_{0 \overline{0}}-\frac{i}{2} \operatorname{Re} \Gamma_{0 \overline{0}}}\right\}+i \frac{\operatorname{Im} A_{0}}{\operatorname{Re} A_{0}}
\end{aligned}
$$

The absorptive part, given by the energy conserving matrix $\Gamma_{i j}$, is formed from products of first-order weak amplitudes which are now the targets of increasingly precise lattice calculations, see for example Refs. [1] and [2]. The dispersive part, $M_{i j}$ is intrinsically second order in $G_{F}$ and contains both a short-distance part were both exchanged $W$ 's are separated by distance scales much smaller than $1 / \Lambda_{\mathrm{QCD}}$ and the long-distance part discussed above.

The computation of the short distance contributions to $\varepsilon_{K}$ or $m_{K_{S}}-m_{K_{L}}$ is now a highly successful application of lattice QCD. At the scale of QCD, phenomena taking place at a distance scale of $1 / m_{W}$ can be accurately represented by an effective four-Fermi operator whose coefficient can be computed in electro-weak perturbation theory and whose matrix element between kaon states can be computed using lattice QCD. For $\varepsilon_{K}$ these short-distances dominate and the long-distance part is estimated to be a few percent correction [3]. However, current results for this short contribution to $\varepsilon_{K}$ are now accurate on the $5 \%$ level, giving the unknown long-distance part increasing importance. 
For the $K_{L}-K_{S}$ mass difference the size of the long-distance contribution is less certain and could provide as much as $50 \%$ of the full result.

It is the lattice calculation of these long distance parts which is the subject of this talk. We will briefly review the approach presented in Ref. [4] for the calculation of the long-distance contribution to the $K_{L}-K_{S}$ mass difference and then describe its generalization to the more complex case of $\varepsilon_{K}$.

\section{Strategy for lattice calculation $K_{L}-K_{S}$ mass difference.}

As presented in Ref. [4] a Euclidean-space, finite volume calculation of the $K_{L}-K_{S}$ mass difference $\Delta m_{K}$ contains three important ingredients. The first is a relation between the second-order, infinite-volume, principal part integral in Eq. 1.3 giving $\Delta m_{K}=2 \operatorname{Re}\left(M_{\overline{0} 0}\right)$ and a finite-volume, discrete perturbation theory sum. This relation is obtained through a generalization of the first-order formula of Lellouch and Luscher for the $K \rightarrow \pi \pi$ decay width [5]. The second ingredient is a computational strategy for evaluating that perturbation theory sum using lattice QCD. While with sufficient statistics the resulting lattice calculation will correctly determine the long-distance part of $\Delta m_{K}$, the short distance contribution to this result will be incorrect, reflecting the details of the lattice regulator. Thus, the third ingredient is a method to replace this erroneous short-distance contribution with the correct short distance part of the continuum theory. We will now briefly review each of these in turn.

The relation between $\Delta m_{K}$ and a finite volume perturbation theory sum follows from the Luscher finite-volume quantization condition [6] relating the allowed finite volume energies, $E_{n}$, for a two-pion system and the $\pi-\pi$ scatting phase shift:

$$
\phi\left(k_{n} L / 2 \pi\right)+\delta_{0}\left(E_{n}\right)+\delta_{W}\left(E_{n}\right)=n \pi
$$

where $k_{n}=\sqrt{E_{n}^{2} / 4-m_{\pi}^{2}}$ and the known function $\phi(y)$ is defined in Ref. [6]. Here we have divided the $\pi-\pi$ scatting phase shift into the strong $s$-wave phase shift $\delta_{0}$ and that caused by the resonant scattering through the weakly coupled $K_{S}$ state. Since the location of the kaon pole in $\delta_{W}\left(E_{n}\right)$ is shifted by $\Delta m_{K}$, Eq. 2.1 can be used to relate $\Delta m_{K}$ to the finite-volume, second-order perturbation theory sum which determines $E_{n}$. It is this argument which we will generalize to determine $\operatorname{Im}\left(M_{\overline{0} 0}\right)$.

Since the resulting finite volume sum that must be evaluated to determine $\Delta m_{K}$ is closely related to perturbation theory, the entire sum can be obtained from an integrated Green's function constructed from four operators: two interpolating operators which create an initial $K^{0}$ state at a time $t_{i}$ and destroy a final $\bar{K}^{0}$ state at $t_{f}$ and two $\Delta S=1$ weak operators $H_{W}\left(t_{1}\right)$ and $H_{W}\left(t_{2}\right)$ evaluated at intermediate times and integrated over a range $t_{f} \gg t_{b} \geq t_{k} \geq t_{a} \gg t_{i}$ for $k=1,2$. The term proportional to length of the integration interval, $t_{b}-t_{a}$ gives the desired perturbation theory sum which determines $\Delta m_{K}$. While the evaluation of this time-integrated four-point function poses substantial computational challenges, our first exploratory study gives encouraging results [7].

Since $\Delta m_{K}$ receives both short- and long-distance contributions, the integrated amplitude described in the previous paragraph will receive important contributions from the region in which the two weak vertices coincide. If a charm quark is included to exploit GIM cancelation a substantial, incorrect short-distance contribution will remain, of $\operatorname{size} \ln \left(m_{W} a\right)$. Fortunately, the same 
techniques which allow this continuum short-distance part to be precisely defined and evaluated in a lattice calculation can be employed here to isolate and replace this lattice artifact by the correct continuum piece. If the four quarks making up the two kaon interpolating operators in the above four-point function are instead treated separately and the resulting Green's function evaluated in Landau gauge and given large, off-shell momentum the short distance contribution can be evaluated numerically in a fashion that is free of infrared singularity allowing a simple substitution by the correct continuum short distance part. Again, first numerical experiments [7] suggest that this can be done without difficulty.

\section{Extension to $\varepsilon_{K}$}

We now turn to the central topic of this talk, the extension of the method reviewed above to the case of $\operatorname{Im}\left(M_{\overline{0} 0}\right)$ and $\varepsilon_{K}$. Since there are no apparent added difficulties associated with the numerical evaluation of the resulting finite-volume expression or the correction of its short-distance part these topics will not be discussed further. However, on first-sight using a Lellouch-Luscher style argument to obtain a finite volume formula for $\operatorname{Im}\left(M_{0 \overline{0}}\right)$ may seem unlikely to succeed. First $\varepsilon_{K}$ describes the mixing of the $K_{S}$ and $K_{L}$ states and the $K_{L}$ state with its predominate decay into three pions would appear inaccessible to Luscher's formalism which applies only to two-particle scattering. Further the energies that are the basis of the Lellouch-Luscher method are intrinsically $\mathrm{CP}$ conserving suggesting no sensitivity to the CP violating phase of $M_{0 \overline{0}}$.

Fortunately both of these problems can be avoided by adding a fictitious, $\Delta S=2$ "superweak" term to the Hamiltonian whose effects can be combined with (but will not alter at leading order) those of the standard weak interactions:

$$
H^{\mathrm{SW}}=\left(\omega_{r}+i \omega_{i}\right)\left\{\bar{s}\left(1+\gamma^{5}\right) \gamma^{\mu} d \bar{s}\left(1+\gamma^{5}\right) \gamma^{\mu} d\right\}+\text { hermitian conjugate. }
$$

The quantities $\omega_{r}$ and $\omega_{i}$ are then chosen so that

$$
\begin{gathered}
\omega_{r}\left\langle\bar{K}^{0}\left|O_{L L}\right| K^{0}\right\rangle+\operatorname{Re} M_{\overline{0} 0}=0 \\
\omega_{i}\left\langle\bar{K}^{0}\left|O_{L L}\right| K^{0}\right\rangle \gg\left|\Gamma_{i j}\right|,
\end{gathered}
$$

for all $i$ and $j$. Here $O_{L L}$ is the operator within the curly brackets in Eq. 3.1. With the off-diagonal, real part of $M_{i j}$ canceled and the off-diagonal term proportional to $\omega_{i}$ dominating the off-diagonal parts of $\Gamma_{i j}$, the eigenstates of the time development operator in Eq. 1.1 become the unfamiliar combinations $K_{ \pm}=\left(\left|K^{0}\right\rangle \pm i\left|\bar{K}^{0}\right\rangle\right) / \sqrt{2}$ — both states which decay predominantly into two pions, consistent with Luscher's finite volume formalism. In addition, the large contribution of $\omega_{i}$ insures that the two $\pi-\pi$ resonances associated with the states $K_{ \pm}$are non-overlapping and will each contribute independent resonant behavior to that scattering. The eigenvalues of the $2 \times 2$ time development matrix on the right-hand side of Eq. 1.1 for the states $\left|K_{ \pm}\right\rangle$can be written:

$$
\begin{aligned}
\lambda_{ \pm}= & \frac{1}{2}\left(M_{00}+M_{\overline{00}}\right) \mp\left(\omega_{i}\left\langle K^{0}\left|O_{L L}\right| \bar{K}^{0}\right\rangle+\operatorname{Im} M_{0 \overline{0}}\right) \\
& +i \frac{1}{4}\left(\Gamma_{00}+\Gamma_{\overline{00}} \mp i \Gamma_{\overline{0} 0} \pm i \Gamma_{0 \overline{0}}\right) \\
= & M_{00} \mp\left(\omega_{i}\left\langle K^{0}\left|O_{L L}\right| \bar{K}^{0}\right\rangle+\operatorname{Im} M_{0 \overline{0}}\right)+i \frac{1}{2}\left(\Gamma_{00} \mp \operatorname{Im} \Gamma_{0 \overline{0}}\right)
\end{aligned}
$$


We first examine the finite volume problem. Here the box size $L$ is adjusted so that we have three nearly degenerate states: the $\pi-\pi$ state $\left|n_{0}\right\rangle$ with energy $E_{n_{0}}$ as well as the two single-particle states, $\left|K^{0}\right\rangle$ and $\left|\bar{K}^{0}\right\rangle$. Using second order, degenerate perturbation theory, we can determine the finite volume energies of interest as the eigenvalues of the $3 \times 3$ matrix:

$$
\left(\begin{array}{ccc}
m_{K}+\sum_{n \neq n_{0}} \frac{\left|\left\langle n\left|H_{W}\right| K^{0}\right\rangle\right|^{2}}{m_{K}-E_{n}} & \sum_{n \neq n_{0}} \frac{\left\langle K^{0}\left|H_{W}\right| n\right\rangle\left\langle n\left|H_{W}\right| \bar{K}^{0}\right\rangle}{m_{K}-E_{n}}+\delta m & \left\langle K^{0}\left|H_{W}\right| n_{0}\right\rangle \\
\sum_{n \neq n_{0}} \frac{\left\langle\bar{K}^{0}\left|H_{W}\right| n\right\rangle\left\langle n\left|H_{W}\right| K^{0}\right\rangle}{m_{K}-E_{n}}+\delta m^{*} & m_{K}+\sum_{n \neq n_{0}} \frac{\left|\left\langle n\left|H_{W}\right| \bar{K}^{0}\right\rangle\right|^{2}}{m_{K}-E_{n}} & \left\langle\bar{K}^{0}\left|H_{W}\right| n_{0}\right\rangle \\
\left\langle n_{0}\left|H_{W}\right| K^{0}\right\rangle & \left\langle n_{0}\left|H_{W}\right| \bar{K}^{0}\right\rangle & E_{n_{0}}+\sum_{n \neq K^{0}, \bar{K}^{0}} \frac{\left.|n n| H_{W}\left|n_{0}\right\rangle\right|^{2}}{E_{n_{0}}-E_{n}}
\end{array}\right),
$$

with the rows and columns corresponding to $\left|K^{0}\right\rangle,\left|\bar{K}^{0}\right\rangle$ and $\left|n_{0}\right\rangle$ in increasing order. Here the complex quantity $\delta m$ is given by:

$$
\delta m=\left(\omega_{r}+i \omega_{i}\right)\left\langle K^{0}\left|O_{L L}\right| \bar{K}^{0}\right\rangle .
$$

Using the $\mathrm{CP}$ and time-reversal operations, the $3 \times 3$ matrix in Eq. 3.6 can be written as:

$$
\left(\begin{array}{ccc}
E_{d 1} & \mathscr{M} & e^{-i \delta_{0}} \mathscr{A} \\
\mathscr{M}^{*} & E_{d 1} & e^{-i \delta_{0} \mathscr{A}^{*}} \\
e^{i \delta_{0}} \mathscr{A}^{*} & e^{i \delta_{0} \mathscr{A}} & E_{d 2}
\end{array}\right) .
$$

The eigenvalues of this matrix can be determined by diagonalizing the large part of the matrix proportional to the first-order amplitude $\mathscr{A}$ and then applying perturbation theory to diagonalize the remainder to order $H_{W}^{2}$ :

$$
\begin{aligned}
E_{0} & =E_{d 1}-\frac{\operatorname{Re}\left\{\mathscr{M}\left(\mathscr{A}^{*}\right)^{2}\right\}}{|\mathscr{A}|^{2}} \\
E_{ \pm} & =\frac{1}{2}\left(E_{d 1}+E_{d 2}\right) \pm \sqrt{2}|\mathscr{A}|+\frac{\operatorname{Re}\left\{\mathscr{M}\left(\mathscr{A}^{*}\right)^{2}\right\}}{2|\mathscr{A}|^{2}} .
\end{aligned}
$$

We now require that the Luscher finite volume condition, Eq. 2.1, be obeyed when the total $\pi-\pi$ phase shift, written as a combination of the usual strong $s$-wave phase shift, the resonant scattering through the $\left|K_{ \pm}\right\rangle$states and a standard second-order-weak Born term is evaluated at the finite-volume energy eigenvalues $E_{ \pm}$given in Eq. 3.10:

$$
\begin{array}{r}
n \pi=\phi\left(\frac{k_{ \pm} L}{2 \pi}\right)+\delta_{0}\left(E_{ \pm}\right)+\arctan \left(\frac{\Gamma_{+}\left(E_{ \pm}\right) / 2}{M_{+}-E_{ \pm}}\right)+\arctan \left(\frac{\Gamma_{-}\left(E_{ \pm}\right) / 2}{M_{-}-E_{ \pm}}\right) \\
-\pi \sum_{\beta \neq K^{0}, \bar{K}^{0}} \frac{\left|\left\langle\beta\left|H_{W}\right| \pi \pi(E)\right\rangle\right|^{2}}{E-E_{\beta}} .
\end{array}
$$

Here $M_{ \pm}$and $\Gamma_{ \pm}$can be determined from the real and imaginary parts of the infinite-volume eigenvalues given in Eq. 3.5:

$$
\begin{aligned}
M_{ \pm} & =m_{K}+M_{00} \mp\left(\omega_{i}\left\langle K^{0}\left|O_{L L}\right| \bar{K}^{0}\right\rangle+\operatorname{Im} M_{0 \overline{0}}\right) \\
\Gamma_{ \pm} & =\Gamma_{00} \mp \operatorname{Im} \Gamma_{0 \overline{0}} .
\end{aligned}
$$

If the finite volume is adjusted so that the zero-order $\pi-\pi$ energy $E_{n_{0}}=m_{K}$, then to zeroth order in $H_{W}$, Eq. 3.11 reduces to the original Luscher quantization condition relating the strong 
$s$-wave phase shift $\delta_{0}\left(E_{ \pm}\right)$and the finite-volume $\pi-\pi$ energy $E_{n_{0}}$. If, for the same value of $E_{n_{0}}$, Eq. 3.11 is expanded to first order in $H_{W}$ then the Lellouch-Luscher relation between the finite volume $K-\pi \pi$ matrix element $\mathscr{A}$ and the $K \rightarrow \pi \pi$ width $\Gamma_{00}$ is obtained.

Considerably more algebra is needed to obtain a relation between the finite volume sum in $\mathscr{M}$ and the infinite volume amplitude $M_{0 \overline{0}}$. As in the earlier study of $\Delta m_{K}$, we first evaluate Eq. 3.11 for a volume which makes the $\pi-\pi$ energy $E_{n_{0}}$ close to $m_{K}$ on the scale of QCD but sufficiently different that a perturbative expansion in $H_{W} /\left(E_{n_{0}}-m_{K}\right)$ is possible. Equation. 3.11 can then be evaluated in a power series in $H_{W}$. The resulting formula contains a pole at $E_{n_{0}}=m_{K}$. Requiring that this pole term vanish gives the standard Lellouch-Luscher relation. Requiring that the non-pole piece vanishes at $E_{n_{0}}=m_{K}$ then relates the infinite-volume, second-order Born term in Eq. 3.11 and the finite-volume, second-order sum in the lower left corner of the matrix in Eq. 3.6.

In the next step, Eq. 3.11 is evaluated for a volume making the two-pion energy $E_{n_{0}}=m_{K}$ and the result expanded to second order in $H_{W}$, including the resonant denominators. The expression that results contains denominators of order $H_{W}^{2}$ and numerators of order $H_{W}^{4}$ and can be written:

$$
\begin{aligned}
0= & \frac{\partial\left(\phi+\delta_{0}\right)}{\partial E}\left\{\sum_{n \neq n_{0}} \frac{\left|\left\langle n\left|H_{W}\right| K^{0}\right\rangle\right|^{2}}{m_{K}-E_{n}}-M_{00}\right. \\
& \left.+\frac{1}{|\mathscr{A}|^{2}} \operatorname{Re}\left(\left(\mathscr{A}^{*}\right)^{2}\left\{\sum_{n \neq n_{0}} \frac{\left\langle K^{0}\left|H_{W}\right| n\right\rangle\left\langle n\left|H_{W}\right| \bar{K}^{0}\right\rangle}{m_{K}-E_{n}}-M_{0 \overline{0}}\right\}\right)\right\} \\
& +\frac{\partial^{2}\left(\phi+\delta_{0}\right)}{\partial E^{2}}|\mathscr{A}|^{2}-2 \frac{\partial}{\partial E_{n_{0}}}\left\{\frac{\partial\left(\phi+\delta_{0}\right)}{\partial E}\left|\left\langle n_{0}\left|H_{W}\right| K^{0}\right\rangle\right|^{2}\right\},
\end{aligned}
$$

giving a finite volume expression for a combination of the infinite volume amplitudes $M_{00}$ and $M_{0 \overline{0}}$.

To determine $M_{0 \overline{0}}$ alone, a second equation is needed. As in the simpler case examined Ref. [4], a second relation follows is we require that the expectation value of the matrix $M_{i j}$ for the state $|\widetilde{K}\rangle=\left(\mathscr{A}\left|K^{0}\right\rangle-\mathscr{A}^{*}\left|\bar{K}^{0}\right\rangle\right) /\left(2|\mathscr{A}|^{2}\right)$ agree with the corresponding finite volume expression up to terms exponentially small in the size of that volume. Here the state $|\widetilde{K}\rangle$ is chosen so that it does not couple to the two-pion, on-shell state, justifying the neglect of finite volume effects. The resulting equation can be combined with Eq. 3.14 to eliminate $M_{00}$ giving the relation:

$$
\begin{aligned}
0= & \operatorname{Re}\left\{( \mathscr { A } ^ { * } ) ^ { 2 } \left(\sum_{n \neq n_{0}} \frac{\left\langle K^{0}\left|H_{W}\right| n\right\rangle\left\langle n\left|H_{W}\right| \bar{K}^{0}\right\rangle}{m_{K}-E_{n}}-M_{0 \overline{0}}\right.\right. \\
& \left.\left.+\frac{1}{\frac{\partial\left(\phi+\delta_{0}\right)}{\partial E}}\left[\frac{1}{2} \frac{\partial^{2}\left(\phi+\delta_{0}\right)}{\partial E^{2}} \mathscr{A}^{2}-\frac{\partial}{\partial E_{n_{0}}}\left\{\frac{\partial\left(\phi+\delta_{0}\right)}{\partial E}\left\langle K^{0}\left|H_{W}\right| n_{0}\right\rangle\left\langle n_{0}\left|H_{W}\right| \bar{K}^{0}\right\rangle\right\}\right]\right)\right\} .
\end{aligned}
$$

Since the $K \rightarrow \pi \pi$ amplitude $\mathscr{A}$ is non-zero with a phase that is independent of the phase of the amplitude in curved brackets, $(\ldots)$, we can conclude that $(\ldots)=0$, our desired finite-volume expression for $M_{0 \overline{0}}$. The real part of this equation gives the earlier result for $\operatorname{Re}\left\{M_{0 \overline{0}}\right\}$. The imaginary part gives the new result for $\operatorname{Im}\left\{M_{0 \overline{0}}\right\}$ :

$$
\begin{gathered}
\operatorname{Im}\left\{M_{0 \overline{0}}\right\}=\operatorname{Im}\left\{\sum_{n \neq n_{0}} \frac{\operatorname{Im}\left\{\left\langle K^{0}\left|H_{W}\right| n\right\rangle\left\langle n\left|H_{W}\right| \bar{K}^{0}\right\rangle\right\}}{m_{K}-E_{n}}+\frac{1}{\frac{\partial\left(\phi+\delta_{0}\right)}{\partial E}}\left[\frac{1}{2} \frac{\partial^{2}\left(\phi+\delta_{0}\right)}{\partial E^{2}}\left\langle K^{0}\left|H_{W}\right| n_{0}\right\rangle\left\langle n_{0} \mid H_{W} \bar{K}^{0}\right\rangle\right.\right. \\
\left.\left.-\frac{\partial}{\partial E_{n_{0}}}\left\{\frac{\partial\left(\phi+\delta_{0}\right)}{\partial E}\left\langle K^{0}\left|H_{W}\right| n_{0}\right\rangle\left\langle n_{0} \mid H_{W} \bar{K}^{0}\right\rangle\right\}\right]\right\} .
\end{gathered}
$$


Thus, by exploiting the interference between the complex, CP-violating amplitude $\mathscr{A}$ and the second-order, Wigner-Weisskopf mass matrix, we have been able to determine the CP-violating part of $M_{0 \overline{0}}$ from a finite-volume energy which is intrinsically CP-even.

Here the left hand side of Eq. 3.16 is the infinite volume quantity which determines the longand short-distance dispersive parts of $\varepsilon_{K}$. The right-hand side contains only finite volume matrix elements which can be evaluated in a lattice QCD calculation. The similarity of the results for $\operatorname{Re}\left\{M_{0 \overline{0}}\right\}, \operatorname{Im}\left\{M_{0 \overline{0}}\right\}$ and $M_{00}$ (not displayed) suggests that a more direct derivation may be possible.

\section{Conclusion}

Given the control over the singularity associated with the principal part and related finite volume effects implied by these results and those in Ref. [4], both the $K_{L}-K_{S}$ mass difference $\Delta m_{K}$ and the complete, dispersive part of $\varepsilon_{K}$ can in principle be computed using lattice methods. In fact, the first exploratory results of Jianglei Yu, presented in Ref. [7], suggest that such a lattice calculation may be possible with the next generation of high performance computers.

However, many obstacles must be overcome: (a) This initial work suggests that the shortdistance parts of $\Delta m_{K}$ and $\varepsilon_{K}$ can be properly treated by a single subtraction, defined using RomeSouthampton techniques. Such a single subtraction is adequate only if GIM cancelation has been realized in the lattice calculation, requiring the inclusion of the charm quark which in turn requires a small lattice spacing. (b) Many more operators and contractions must be included in a complete calculation than have been attempted in Ref. [7]. (c) These initial calculations use a relatively massive, $410 \mathrm{MeV}$ pion. The successful subtraction of an exponentially growing single-pion contribution demonstrated in Ref. [7] will become more difficult as the pion mass is reduced. Even greater difficulty will result from the vacuum subtraction needed when disconnected graphs are included. We believe that these difficulties can be overcome with improved numerical methods and the sustained, 100 Tflops capability of the next generation machines now being installed. The author acknowledges the important contributions of his RBC/UKQCD collaborators to this work.

\section{References}

[1] T. Blum, P. Boyle, N. Christ, N. Garron, E. Goode et al., $K$ to $\pi \pi$ Decay amplitudes from Lattice QCD, arXiv:1106.2714 [hep-lat].

[2] T. Blum, P. Boyle, N. Christ, N. Garron, E. Goode et al., The $K \rightarrow(\pi \pi)_{I=2}$ Decay Amplitude from Lattice $Q C D$, arXiv:arXiv:1111.1699 [hep-lat] .

[3] A. J. Buras, D. Guadagnoli and G. Isidori, On $\varepsilon_{K}$ beyond lowest order in the Operator Product Expansion, Phys. Lett. B688 (2010) 309-313 [arXiv:1002.3612 [hep-ph] ].

[4] RBC and UKQCD Collaboration, N. H. Christ, Computing the long-distance contribution to second order weak amplitudes, PoS LATTICE2010 (2010) 300 [arXiv: 1012.6034 [hep-lat] ].

[5] L. Lellouch and M. Luscher, Weak transition matrix elements from finite-volume correlation functions, Commun. Math. Phys. 219 (2001) 31-44 [hep-lat/0003023].

[6] M. Luscher, Two particle states on a torus and their relation to the scattering matrix, Nucl. Phys. $\mathbf{B 3 5 4}$ (1991) 531-578.

[7] J. Yu, Long distance contribution to $K_{L}-K_{S}$ mass difference, arXiv:1111.6953 [hep-lat ]. 